\title{
Adverbiaux initiaux : perspectives en français et en finnois
}

Clause-initial adverbials: perspectives in French and Finnish

Lauseenalkuiset adverbiaalit : näkökohtia ranskassa ja suomessa

\section{Soili Hakulinen et Meri Larjavaara}

\section{(2) OpenEdition}

Journals

Édition électronique

URL : https://journals.openedition.org/efo/4194

DOI : 10.4000/efo.4194

ISSN : 2275-1947

\section{Éditeur}

INALCO

Édition imprimée

Date de publication : 1 janvier 2014

ISBN : 978-2-343-05394-3

ISSN : 0071-2051

\section{Référence électronique}

Soili Hakulinen et Meri Larjavaara, "Adverbiaux initiaux : perspectives en français et en finnois », Études finno-ougriennes [En ligne], 46 | 2014, mis en ligne le 15 octobre 2015, consulté le 08 juillet 2021. URL : http://journals.openedition.org/efo/4194 ; DOI : https://doi.org/10.4000/efo.4194

Ce document a été généré automatiquement le 8 juillet 2021.

\section{(c) (7) (8)}

Études finno-ougriennes est mis à disposition selon les termes de la Licence Creative Commons Attribution - Pas d'Utilisation Commerciale 4.0 International. 


\title{
Adverbiaux initiaux : perspectives en français et en finnois
}

\author{
Clause-initial adverbials: perspectives in French and Finnish \\ Lauseenalkuiset adverbiaalit : näkökohtia ranskassa ja suomessa
}

Soili Hakulinen et Meri Larjavaara

Nous remercions Luciane Hakulinen d'avoir bien voulu relire ce texte, ainsi que les deux relecteurs anonymes de leurs remarques pertinentes.

\section{Introduction}

1 Le point de départ pour cette étude a été l'idée basée sur l'intuition - pour ne pas dire l'idée naïve - que le statut des circonstants placés en début de phrase serait très différent en français et en finnois écrits. Nous avons été confrontées à cette question lors de notre enseignement de la linguistique et parallèlement de la langue française dans les universités finlandaises.

2 Selon notre première hypothèse, les circonstants initiaux seraient plus fréquents en français et cet emplacement ne serait pas dû à une motivation particulière, tandis qu'en finnois la position en début de phrase serait toujours marquée (notre hypothèse ne nous disait pas encore comment et pourquoi). L'ordre des constituants dans les exemples (1) et (2) serait donc naturel selon notre hypothèse ; l'ordre dans (3) serait possible mais marqué et demanderait donc des conditions spéciales (dans nos exemples le circonstant observé est souligné) :

(1) Souvent elle doit partir à $7 \mathrm{~h}$.

(2) Hän joutuu usein lähtemään 7 :ltä.

(3) Usein hän joutuu lähtemään 7 :ltä.

3 Avec cette hypothèse à l'esprit, nous sommes parties à la recherche d'occurrences. Notre objectif était de travailler sur des corpus comparables dans les deux langues et d'y analyser tous les circonstants placés au début d'une proposition définie selon des 
critères morphosyntaxiques et typographiques. Il s'agira ensuite de comparer les observations faites, pour les deux langues, dans un cadre fonctionnel (dans le sens moderne du terme).

Avant de commencer, il convient de mentionner l'étude de Välikangas (1984), intitulée «Les compléments circonstanciels initiaux dans les propositions finnoises et françaises", qui est une première ébauche de typologie de circonstants initiaux. Välikangas passe en revue les différents types de circonstants initiaux et veut trouver des paramètres pour une comparaison syntaxique du finnois et du français. L'objectif de son étude ne correspond donc pas au but du présent travail.

\section{Corpus}

Nous avons naturellement considéré comme important d'avoir des matériaux comparables pour les deux langues et avons voulu travailler avec des corpus parallèles. Pour ce dessein, la solution la plus accessible semblait être de choisir deux romans, l'un en français et l'autre en finnois, et d'utiliser ces textes ainsi que leurs traductions respectives. Notre choix a porté sur deux romans récents qui ont une narration plutôt classique : Dans les bois éternels par Fred Vargas et Puhdistus de Sofi Oksanen, ainsi que leurs traductions sous les titres respectifs Ikimetsän sydän et Purge ${ }^{1}$.

6 Nous avons retiré les 100 premières occurrences qui nous intéressaient dans les textes originaux et leur traduction et avons fait une première analyse à partir de ce petit corpus préliminaire. Malheureusement, il nous a semblé d'emblée évident que ce corpus littéraire ne pouvait pas satisfaire les besoins de notre recherche. Tout d'abord, les traductions étaient bien trop proches des textes originaux : l'influence de la langue de départ semblait trop forte pour notre objectif. D'autre part, les textes eux-mêmes ont posé des difficultés : le roman de Vargas contenait beaucoup de dialogues, ce qui rendait la narration fragmentaire, et le début de celui d'oksanen était un monologue intérieur du personnage où les mêmes constructions, jusqu'aux mêmes mots, se répétaient.

7 Il nous a donc fallu trouver autre chose, et nous avons décidé de tirer parti d'un corpus rassemblé pour l'étude des chaînes de référence, de Hakulinen \& Turkka (à paraître) ${ }^{2}$. C'est un corpus de textes de presse dont une partie nous semblait parfaite également pour notre étude : nous avons donc analysé le sous-corpus constitué de douze articles de presse en français et de treize articles en finnois, parus dans des quotidiens à statut comparable (Le Monde et Helsingin Sanomat), à la même période (pour la plupart pendant l'hiver 2010-2011). Les articles choisis parlent de personnes connues dans les deux pays (certaines plus connues en France, d'autres en Finlande). Il n'a pas été possible d'avoir une masse de texte comparable dans les deux langues, puisque les articles du Monde sont systématiquement plus longs que ceux de Helsingin Sanomat, et ainsi le corpus finnois contient-il nettement moins de caractères ( $38 \%$ de moins) que le corpus français. C'est sur ce corpus de textes de presse que se construit notre présente analyse.

\section{Pour aboutir à une définition}

8 La catégorie de l'adverbial vs. du circonstant est problématique, et certains sont même prêts à dire que la catégorie entière du circonstant devient inutilisable en grammaire, 
tant sa définition pose de problèmes. Il ne sera pas question ici de contribuer à cette discussion (cf. à ce sujet Rémi-Giraud 1998), mais pour avoir une définition opérationnelle de ce constituant, nous évoquerons deux points qui sont pertinents pour notre étude.

Le premier point concerne le niveau hiérarchique où se situe le constituant en question dans la phrase. Le circonstant est souvent défini comme le troisième constituant majeur, facultatif, de la phrase de base, les deux autres étant le SN sujet et le SV (ex. Riegel et al. 2009, p. 260). Selon cette définition, le circonstant n'est pas un argument du verbe, mais est dominé directement par la phrase dans la structure hiérarchique de la phrase. La caractéristique syntaxique d'un tel constituant est d'être mobile et supprimable: sa suppression ou son déplacement ne rend pas une phrase agrammaticale, comme c'est le cas dans (4) :

(4) Ce jour-là, la députée européenne a donné rendez-vous à la presse aux côtés de son avocat, William Bourdon. (Joly)

Cependant, il existe d'autres constituants non argumentaux, c'est-à-dire facultatifs, mais qui ne sont pas dominés par la phrase, mais par le verbe :

(5) Il chante mal.

(6) En écho, la numéro un des Verts assure qu'elle « ne fera rien contre Eva ». (Joly)

11 Selon l'approche de Riegel et al. (2009, p. 261), les constituants soulignés dans (5) et (6) ne sont pas considérés comme des circonstants, mais comme des compléments du verbe $^{3}$, alors que d'autres approches les assimilent aux circonstants (ex. Rémi-Giraud 1998, p. 82-83). Cependant, la distinction entre ces deux types de constituants ${ }^{4}$ ne s'est pas avérée importante dans cette étude : les constituants facultatifs situés à chacun des deux niveaux hiérarchiques semblent avoir un comportement discursif identique en position initiale. Les deux seront pris en compte dans cette étude.

D'autre part, le circonstant, constituant non essentiel, s'oppose au constituant essentiel du verbe appelé objet (direct ou indirect). Or il est connu que dans bien des cas, il n'est pas facile de distinguer entre objet indirect et circonstant. Il s'agit plutôt d'un continuum entre deux cas de figure prototypiques : à une extrémité se trouve l'objet indirect, et à l'autre, le circonstant. Entre les deux, il y a différents cas qui sont plus ou moins clairs, par exemple, certains circonstants sont plus mobiles que d'autres. L'exemple (7) illustre un cas où il n'est pas évident de trancher :

(7) « Pour quelques-uns, tout est permis alors que le pays est dans une situation dramatique », lance-t-elle à la salle plongée dans un silence attentif. (Joly)

Le constituant souligné est ici mobile et n'est pas introduit par la préposition attendue pour le verbe permettre (permettre à); sémantiquement il semble toutefois s'accommoder parfaitement au verbe et être même exigé par celui-ci.

En linguistique finnoise, le circonstant correspond en gros à l'adverbial (adverbiaali). Les compléments argumentaux du verbe sont, selon la "Grande grammaire finnoise " (Hakulinen et al. 2004, désormais ISK 2004, p. 829), le sujet, l'objet, l'attribut (predikatiivi) et les compléments adverbiaux. Selon la linguistique finnoise, ces derniers sont dans des cas sémantiques. Par cela, on réfère aux cas autres que le nominatif, l'accusatif, le partitif et le génitif5.

15 Aussi bien des compléments régis par le verbe que des compléments moins intimement liés au verbe - les compléments de phrase - sont appelés adverbiaux (id. p. 866). De 
même, pour utiliser cette dénomination, il importe peu de savoir si le complément est essentiel et appartient à la valence du verbe ou non. L'adverbial est un terme qui convient dans tous ces cas.

Il faut encore noter que, même dans le cadre de la linguistique française, certains auteurs ont opté pour le terme adverbial pour désigner de façon générale ces différentes fonctions syntaxiques qui ne sont ni sujet ni objet ni attribut. C'est le cas de Michel Charolles et de Denis Vigier notamment (p.ex., Charolles 2003, Charolles \& Vigier 2005). Il nous semble que ce terme pourrait effectivement être plus souvent utilisé en linguistique française - comme nous l'avons vu, les termes "circonstant» ou " complément circonstanciel » ont des acceptions différentes chez différents auteurs.

Pour récapituler, lors de la collecte du corpus, il s'agissait d'y inclure les phrases qui ont en tête un constituant autre que le sujet, le verbe, l'objet ou l'attribut. Ce constituant est le plus souvent un circonstant, ou quelquefois il peut se rapprocher d'un objet indirect. Nous l'appelons dans ce travail adverbial.

\section{Choix des formes relevées : critère morphologique et positionnel}

La sélection des occurrences a été faite à partir de critères morphologiques et positionnels. En principe, toutes les propositions contenant des constituants obliques situés en tête ont été relevées, avec une restriction cependant : seuls les constituants nominaux font partie de l'étude dans ce premier temps. Ainsi ont été laissés de côté tous les constituants comportant une forme verbale, qu'ils soient à une forme finie ou non finie (propositions subordonnées, infinitifs, participes en emploi verbal, gérondifs, et en finnois les "quasi-propositions", lauseenvastike). La forme morphologique du constituant est dans la grande majorité des cas un SP, mais peut aussi être un SN (ce jour-là), ou une partie simple de discours (adverbe ou connecteur).

19 Ont donc été relevées les propositions introduites par un connecteur ou un modalisateur ( $c f$. Rémi-Giraud 1998, p. 103). Par contre, les conjonctions n'ont pas été prises en compte : non déplaçables, elles ne peuvent occuper que la première place de la proposition.

Le corpus inclut également les prédications secondes, qui introduisent dans la proposition une nouvelle structure prédicative réduite (Combettes 1998). Seules les prédications secondes qui sont formées d'éléments non verbaux ont été relevées (participes passés adjectivaux inclus) :

(8) Mal peignées également, ces chansons turbulentes cherchent à marier l'éloquence poétique et la fièvre primitive du rock. (Smith)

(9) Valtiona Libya eroaa suuresti Egyptistä ja Tunisiasta, joissa yhtenäinen kansallistunto antoi voimaa vastalauseliikkeelle ja auttoi myös viemään asevoimat vähitellen mielenosoittajien rinnalle. (Gaddafi 1) [trad. En tant qu'État, la Libye diffère beaucoup de l'Égypte et de la Tunisie, où un sentiment national unifié a renforcé le mouvement de révolte...]

21 Bien que les prédications secondes ne soient pas à proprement parler des adverbiaux, au plan sémantique elles expriment souvent des relations circonstancielles (RémiGiraud 1998, note 72). De plus, elles sont susceptibles d'avoir un fonctionnement discursif identique aux adverbiaux (Combettes 2005). 
(erikoislausetyypit) pour lesquelles souvent, dans une construction non marquée, un adverbial justement se place en tête. Les phrases existentielles, par exemple, en sont une sous-catégorie (ISK 2004, p. 850-852). Elles sont souvent traduisibles en français par la construction il $y$ a et commencent donc par un locatif :

(10) Levyillä oli liikaa nuotteja, kuvioita, rönsyjä

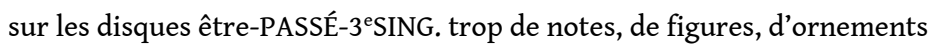

- niin paljon kaikkea, että kuunteleminen kävi voimille. (Jarrett)

[trad. Il y avait trop de notes, de figures, d'ornements dans les disques - tellement de choses qu'il devenait fatigant de les écouter.]

Les phrases possessives, où la traduction française contient le plus souvent le verbe avoir, en sont un autre exemple (11), ou encore les phrases (12) et (13), où l'adverbial du finnois est de même traduit en français comme un sujet (sa position non marquée est sans conteste initiale ; ISK 2004, p. 852-862) :

(11) Sarkozylla oli

Sarkozy-ADESSIF-SING.être-PASSÉ-3eSING.

vakavia ongelmia jo ennen valintaa. (Joly 2)

graves problèmes-PARTITIF-PL. déjà avant l'élection

[trad. Sarkozy avait de graves problèmes déjà avant son élection.] 
(12) Tutkintaan voi kulua

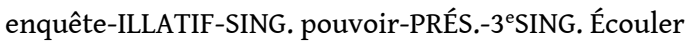

vuosia. (Joly 2)

année-PARTITIF-PL.

[trad. L'enquête peut prendre des années.]

(13) Norjalaisesta au pair -tytöstä tuli

jeune fille au pair norvégienne-ÉLATIF-SING. devenir-PASSÉ-3 ${ }^{\text {eSING }}$

ensin Ranskan tunnetuin rötösherrojen jahtaaja. (Joly 2)

d'abord le plus célèbre chasseur de corrupteurs en France-NOM.-SING.

[trad. La jeune fille au pair norvégienne est d'abord devenue la plus célèbre dénonciatrice de la corruption en France.]

Les phrases de type spécifique du finnois sont difficilement analysables en fonctions syntaxiques traditionnelles. Il s'agit plutôt de les considérer comme des « conglomérats » de différents types de caractéristiques (ISK 2004, p. 849).

Il existe cependant en finnois aussi d'autres phrases où un adverbial a son emplacement le moins marqué en tête de phrase :

(14) Jarrettiin iski viisikymmenvuotiaana sairaus, joka vei voimat niin tyystin, että hän ei jaksanut kääntää välillä edes kirjan sivua. (Jarrett)

[trad. À 50 ans Jarrett a été atteint d'une maladie qui lui a ôté les forces à un tel point qu'il lui arrivait d'avoir du mal à tourner les pages d'un livre.]

(15) Kohu osoittaa, että taustastani on niin vaikea repiä skandaaleja, että lähes kaikki kelpaa. (Duflot)

[trad. Cette rumeur prouve qu'il est si difficile de trouver des scandales dans mon passé que presque n'importe quoi peut faire l'affaire.]

Ces phrases n'entrent pas directement dans les catégories des phrases spécifiques décrites par ISK (2004), mais elles présentent des caractéristiques semblables ou d'un autre type qui jouent pour l'emplacement de l'adverbial au début («les phrases à phénomène » ilmiölause pour 14 , sujet infinitif dans 15 , etc.). De plus, en règle générale, s'il n'y a pas de sujet, un adverbial se place volontiers dans la position de thème (teemapaikka) en début de phrase, et c'est le cas du passif finnois également (ISK 2004, p. 1311) :

(16) [...] jos he luovuttavat, heille kostetaan äärimmäisen julmasti. (Gaddafi 1)

[trad. ...s'ils se rendent, on se vengera d'eux d'une manière extrêmement cruelle.]

Si nous nous intéressons à la motivation de la position initiale des adverbiaux dans les deux langues, il nous faut analyser les cas où cette position est due à un choix opéré par le locuteur en fonction du discours et non aux règles syntaxiques appartenant à la phrase elle-même. Tous les cas présentés dans ce sous-chapitre, bien qu'ils fassent partie de notre corpus et soient intéressants en soi, proposent à l'adverbial un emplacement non marqué au début de la proposition.

Dans notre corpus de textes de presse, ces phrases, où il est clair que la position de l'adverbial en tête de phrase n'est pas marquée, représentent environ $30 \%$ dans le corpus finnois. Si nous laissons ces cas de côté, nous avons à peu près le même nombre de propositions dans les deux langues (114 en finnois, 118 en français). 


\section{Motivations thématiques}

33 Il est clair que la motivation de la présence en tête de phrase de l'adverbial doit se trouver dans des considérations d'ordre informationnel. C'est la structure informationnelle de la phrase - c'est-à-dire l'emplacement du thème - qui se trouve à l'origine de cet ordre des constituants. Le positionnement du thème dans la phrase est fondamental mais n'est cependant pas une explication suffisante dans tous les cas. L'approche textuelle, qui s'étend au-delà de la limite de la phrase, devient indispensable.

\subsection{La notion de thème en linguistique française}

Nous tirons profit de la notion de thème utilisée par Charolles (2003), qui appelle le thème le topique, mais dit explicitement qu'il pourrait aussi bien utiliser le terme thème (Charolles 2003, note 1). Le thème est une notion qui réfère à la structure informationnelle de la phrase : il désigne une fonction qui contient des informations supposées connues, c'est-à-dire accessibles dans le contexte - soit par le contexte langagier, soit par les connaissances extralinguistiques -, et susceptibles de servir de support ou de repère pour des informations nouvelles. Le thème est une fonction qui figure de préférence en tête de proposition ou en zone préverbale, place certainement motivée par la linéarité du langage, étant donné qu'il est naturel de construire le nouveau savoir sur quelque chose de déjà connu.

Dans les langues SVC comme le français ou le finnois, c'est le plus souvent le sujet qui est considéré comme le thème dans une phrase neutre, c'est-à-dire non marquée. En finnois, il faut cependant se souvenir notamment des phrases de «type spécifique » que nous avons déjà discutées. D'autre part, on peut avoir comme thème de la phrase - par excellence ! - un constituant disloqué (constituant qui est détaché en tête de phrase, puis repris par un pronom à l'intérieur de la structure de la phrase : Ta voiture, elle est mal garée). Ces constituants sont des expressions référentielles, pour lesquelles l'idée de thème comme quelque chose de connu s'applique effectivement assez facilement.

Cependant, on peut avoir aussi en tête de phrase un adverbial. Cette position de l'adverbial est-elle, de la même façon, motivée par le fait qu'il est thématique dans la phrase ? Ou y a-t-il une autre raison pour qu'il apparaisse en tête?

Pour que l'adverbial soit considéré comme thème, il faudrait - dans le cas typique qu'il reprenne d'une façon ou d'une autre quelque chose dont il a déjà été question dans le texte, quelque chose de connu. Selon Charolles (2003, p. 8), la thématicité (topicalité) des adverbiaux est néanmoins bien plus difficile à statuer que celle des expressions référentielles, puisque les adverbiaux ne renvoient pas souvent à des référents concrets - à part les adverbiaux locatifs, qui renvoient à des endroits ou des zones de l'espace, qui sont des référents concrets. Les adverbiaux de temps, de manière, de cause, etc., ne renvoyant pas à des référents concrets, restent dans ce sens difficilement classifiables selon les critères de thématicité.

\subsection{La position de thème en linguistique finnoise}

38 En ce qui concerne le finnois, bien que ce soit une langue où l'ordre SVC est le plus fréquent, divers facteurs peuvent faire qu'un ordre linéaire syntaxique autre est senti 
comme non marqué. Comme ce sont les cas qui permettent en finnois de faire varier l'ordre des constituants, celui-ci peut aisément être utilisé pour des fins informationnelles. Notamment, les linguistes ont présenté le modèle positionnel suivant (proposé par Vilkuna 1989; développé p. ex. dans ISK 2004, p. 1306 ; voir également Duvallon 2003 et 2011, d'où nous proviennent les traductions des termes).

\begin{tabular}{|l|l|l|l|l|}
\hline Champ introductif & \multicolumn{2}{|l|}{ Champ initial } & Champ final & Clôture \\
\hline & Position frontale & Positionde thème & & \\
\hline
\end{tabular}

Dans les types de phrase spécifiques, l'élément que nous avions identifié comme adverbial occupait la position de thème.

En général, avant la position de thème, la position frontale peut être occupée par d'autres éléments, par exemple par différents adverbiaux "sans effet de sens particulier » (Duvallon 2011, p. 8-9, l'exemple est d'elle également) :

Position frontale $\rightarrow$ Position de thème $\rightarrow$ Champ final

Seuraavan kahden päivän aikana suru ja viha saostuivat au cours des deux jours suivants le chagrin et la colère épaissir-PASSÉ-3 $3^{\mathrm{P}} \mathrm{PL}$.

[trad. Au cours des deux jours suivants, le chagrin et la colère ont épaissi.]

La plus grande partie de notre corpus est constituée d'adverbiaux du champ initial.

\subsection{La thématisation}

Fournier (1997, p.120) constate pour le français qu'un adverbial (elle parle de complément prépositionnel ou d'adverbe) est mis en tête de phrase lorsqu'il est thématisé, posé comme point de départ connu dans le discours. Selon Fournier, la thématisation peut se faire de deux façons dont l'une, qui s'appelle la thématisation anaphorique, est la reprise du contexte arrière. Dans ce cas, l'adverbial initial construit la progression thématique du texte en reprenant anaphoriquement un élément du contexte gauche, comme l'illustre l'exemple (17) où ce jour-là (souligné) reprend anaphoriquement le 23 juin (en gras) de la phrase précédente :

(17) $\mathrm{M}^{\mathrm{me}}$ Joly - Gro Eva de son prénom complet - continue à dénoncer ce "scandale d'État", réclamant une instruction indépendante. "Vous savez qu'un financement illicite de campagne électorale entraîne l'annulation d'une élection”, se risque-t-elle, le 23 juin, en faisant allusion aux accusations d'enveloppes perçues pour la campagne présidentielle de Nicolas Sarkozy faites par l'ex-comptable de $\mathrm{M}^{\mathrm{me}}$ Bettencourt. Ce jour-là, la députée européenne a donné rendez-vous à la presse aux côtés de son avocat, William Bourdon. (Joly 1)

La thématisation de l'adverbial peut se faire aussi par la construction d'un cadre de référence dans la phrase, cadre dans lequel le contenu de la phrase est ensuite validé. Dans ce cas également, l'adverbial initial devient le point de repère thématique dont part le reste de l'énoncé, mais il n'a pas de relation anaphorique directe avec le contexte précédent et il ne contribue pas à la construction de la progression thématique. L'exemple (18) illustre le cas où l'adverbial initial dans son appartement construit un nouveau cadre de référence à l'énoncé : 
(18) Car la Franco-norvégienne pense qu'il ne faut pas mettre de limite à ses envies, que la vie est pleine de rebondissements possibles: "Tu es secrétaire aujourd'hui mais maîtresse de ton destin. Il n'y a pas de déterminisme. Je déteste qu'on limite son avenir parce qu'on a dépassé 60 ans !", s'enflamme-t-elle soudain. [alinéa] Dans son appartement, elle a longtemps accroché la photo d'une femme d'un certain âge, handicapée par une prothèse de hanche, mais en train de plonger à l'eau : "Elle était magnifique." (Joly)

Cet adverbial initial est également en tête de paragraphe : il n'a pas été question de l'appartement ou d'autre lieu auparavant, celui-ci sert de cadre de référence pour parler d'une nouvelle chose dans le texte.

L'explication de Fournier reste toutefois insuffisante et doit être affinée, et c'est ce qu'ont fait plusieurs chercheurs (entre autres, Virtanen 1992, 2004 ; Charolles 2003 ; Charolles \& Vigier 2005, cf. ci-dessous). L'emplacement de l'adverbial est motivé par plusieurs facteurs et dépend du contexte qui l'entoure, mais aussi bien le contexte gauche que le contexte droit doivent être pris en compte. Nous allons envisager à présent, dans une perspective contrastive français-finnois, quatre motivations principales accompagnées de quelques sous-catégories qui peuvent entraîner l'emplacement initial de l'adverbial. Notons que deux ou plusieurs motivations peuvent agir simultanément.

\subsection{Motivation 1 : influence du contexte gauche}

Reprenons : si le locuteur choisit de placer un adverbial en tête de proposition, c'est parce qu'il a une raison pour le faire (cf. Charolles 2003, p. 16) ; le locuteur organise ce qu'il a à dire selon ce qu'il met en première position dans la proposition. Il y a toujours une motivation particulière pour cette position marquée.

Si cette motivation se trouve dans le contexte gauche ou en amont du texte, c'est qu'on a parlé auparavant de l'information reprise par l'adverbial. Il s'agit d'un thème : un élément dont il a déjà été question dans le texte est repris en tête d'une nouvelle proposition $^{8}$. Il existe alors un lien référentiel qui peut être de différents types vers un élément antérieur du texte. La plupart des adverbiaux initiaux analysés dans les textes journalistiques français reprennent un élément du discours antérieur (72\%), et il en va de même avec le corpus journalistique finnois (65\%).

lexemple (19), le lien anaphorique est évident. On parle du voyage en avion de Kadhafi (Kadhafi s'envole pour Bruxelles) et même du tapis rouge, et après l'alinéa, on commence le nouveau paragraphe par à son arrivée. Il y a ici un rapport direct avec ce qui précède :

(19) Juste après l'ouverture de l'ambassade des États-Unis à Tripoli, Kadhafi s'envole pour Bruxelles où on lui déroule le tapis rouge. [alinéa] À son arrivée, il déclare que la Libye est déterminée et engagée à jouer un rôle de chef de file pour arriver à la paix dans le monde. (Kadhafi 4)

50 Le lien référentiel peut parfois être très clair même s'il n'y a pas de rapport strictement anaphorique ( $c f$. anaphore indirecte) :

(20) Cécile Duflot a pris son temps avant de répondre à l'“Appel du 22 mars" de Daniel Cohn-

Bendit. Dans un courrier qu'elle adresse, lundi 12 avril, à tous les militants Verts, comme à ceux d'Europe Ecologie, la secrétaire nationale des Verts donne sa vision de l'avenir des écologistes.

(Duflot 1) 
51

Ou, exemple du finnois :

(21) Puolueelle valitaan uusi puheenjohtaja ensi syksynä tai alkuvuonna 2011. Paikasta kisaa Marine Le Penin kanssa todennäköisesti FN :n varapuheenjohtaja Bruno Gollnisch. (Le Pen 1) [trad. Un nouveau secrétaire général sera choisi pour le parti l'automne prochain ou au début de l'année 2011. Bruno Gollnisch, le vice-président du FN, sera probablement en concurrence avec Marine Le Pen pour ce poste.]

Il existe néanmoins de légères différences entre les deux langues dans la mesure où le finnois accepte plus de répétition. En finnois, un même mot peut être répété tel quel pour bien mettre en évidence le lien référentiel :

(22) EU :n yhteinen verotusjärjestelmä on Jolyn haave. Haaveeksi se saattaa jäädäkin, koska jäsenmaat eivät hevin luovu omista verolaeistaan. (Joly 2) [trad. Le rêve de Mme Joly est une imposition commune dans toute l'Europe. Cela peut rester (à l'état de) rêve parce que les États membres ne renoncent pas facilement à leur législation fiscale.]

De plus, les deux mots se suivent ici directement dans le texte.

Ce type de reprise est, pour des raisons stylistiques, rare en français et inexistant dans notre corpus français, mais facilement trouvable dans des textes finnois, où il est justement utilisé pour mettre l'accent sur l'élément thématique. En français, on a tendance à éviter la répétition inchangée et immédiate du syntagme nominal, mais si celui-ci est différemment déterminé et modifié, la répétition devient possible (ici, les deux occurrences de discours) :

(23) “Je me battrai jusqu'à la dernière goutte de mon sang". C'est par des mots très durs que Mouammar Kadhafi s'est adressé à son peuple, mardi 22 février, dans son premier discours en direct à la télévision depuis le début des manifestations réclamant son départ.

Dans ce discours, long, confus et véhément, le dictateur libyen a assuré qu'il n'était pas question de quitter le pouvoir comme l'ont fait d'autres dirigeants du monde arabe. (Kadhafi 1)

Quant à la distance linéaire dans le texte, l'élément repris se trouve presque toujours dans la phrase précédente, mais ceci n'est pas une règle absolue : il peut également être situé à une distance de plusieurs phrases, voire de plusieurs paragraphes de la phrase avec l'adverbial initial, comme c'est le cas dans les deux exemples suivants :

(24) Les adhérents du FN ont eu environ un mois pour voter par correspondance. Les résultats doivent être proclamés officiellement dimanche. La veille, Jean-Marie Le Pen aura tenu son dernier discours en tant que président du FN, au premier jour du XIV congrès de la formation politique qui se déroule à Tours. [alinéa ; 5 lignes de texte ; alinéa] Pendant la durée du vote qui a couru sur décembre 2010 et le mois de janvier, $\mathrm{M}^{\text {me }}$ Le Pen a été au centre du débat politique après ses déclarations rapprochant les prières musulmanes dans la rue et l'Occupation. (Le Pen 2)

(25) Aggressiivisessa puheessaan Gaddafi uhkasi nujertaa mielenosoittajat kuin torakat. Uhkaus oli pelästyttänyt mielenosoittajat. [5 paragraphes courts ; alinéa] Gaddafille uskolliset joukot pienenivät yhä keskiviikkona. Tiistain puheen jälkeen tuhannet Gaddafin kannattajat siirtyivät Tripolin keskusaukiolla mielenosoittajien joukkoon, NYT kertoi. (Gaddafi 4) [trad. Dans son discours agressif, Kadhafi a menacé d'écraser les manifestants comme des cafards. La menace avait fait peur aux manifestants. [ 5 paragraphes courts ; alinéa] Les troupes fidèles à Kadhafi ont encore diminué mercredi. Après le discours de mardi, des milliers de partisans de Kadhafi ont rejoint les manifestants sur la place centrale de Tripoli, écrit The New York Times.]

La thématicité, reprenant un élément du discours antérieur, est donc une caractéristique fréquente d'un adverbial initial. L'utilisation d'un adverbial initial participe à la construction de la cohésion textuelle dans les deux langues. 


\subsection{Motivation 1b : Fonction contrastive} particulière : celui-ci occupe alors une position contrastive par rapport à un autre élément dans la phrase ou dans le texte. Il ne reprend alors pas directement un thème, mais un élément $\mathrm{du}$ contexte précédent est repris comme repère pour un positionnement qui contraste avec ce qui est déjà dit. Le lien cohésif avec le contexte gauche appartient alors au plan sémantique et se traduit par l'introduction d'un élément en contraste avec quelque chose qui précède. Cette situation, qu'à notre connaissance on ne commente pas dans les grammaires françaises, revient à la position frontale du finnois (esikenttä). Cette position est réservée à un thème changeant (vaihtuva teema : ISK 2004, p. 1309, 1318). l'on peut donc parler d'un thème en contraste :

(26) J'ai commencé en 1990, mais soudain ma vie a de nouveau basculé dans le malheur. (Smith)

(27) Je suis même venue manifester à Paris puisqu'aux États-Unis on ne faisait rien. (Smith 1)

(28) Toujours aussi ardente, mais meilleure chanteuse qu'en 1975, Patti Smith y rayonne plus que jamais. "À l'époque, nous l'appelions notre field marshal, rigole Lenny Kaye. Aujourd'hui, elle est toujours notre commandeur, une artiste multiple qui s'est épanouie avec le temps. »

En finnois également, ce caractère contrastif semble être fréquent (17 \%) :

(29) "[...] Ranskan oikeisto on talouskysymyksissä hyvin liberaali ja monissa muissa asioissa hyvin autoritäärinen. Tämä ei ole yhteensovitettavissa vihreyteen." [alinéa] Sosialistiyhteistyössä hiertää ydinvoima, jota sosialistit kannattavat vahvasti. (Duflot)

[trad. La droite française est très libérale dans les questions de finance mais très autoritaire dans bien d'autres matières. Ceci est contraire à la mentalité des Verts. [alinéa] Dans la collaboration avec les socialistes, c'est le nucléaire qui pose problème, car les socialistes se prononcent fortement pour le nucléaire.]

(30) Se oli myös välttämätöntä, sillä hänellä ei ollut voimia, joita olisi voinut "tuhlata". Hänen oli pakko keskittyä entistä enemmän olennaiseen. [alinéa] "Nykyään ${ }^{9}$ voin oikein hyvin, mutta soolokonsertteja en voi suunnitella pitkälle." (Jarrett)

[trad. C'était aussi nécessaire, car il n'avait pas de forces qu'il aurait pu "gaspiller". Il devait se concentrer de plus en plus sur l'essentiel. [alinéa] « Actuellement je vais très bien, mais je ne peux pas planifier longtemps à l'avance des concerts solos. »]

\section{Le cas des clivées}

60 Le français dispose d'une autre manière de marquer la fonction contrastive d'un constituant placé à l'initiale de la phrase : il peut attirer l'attention sur un constituant avec une structure spécifique, le clivage. Selon Riegel et al. (2009, p. 736), il s'agit d'une opération de focalisation d'un constituant : contrairement à la thématisation, l'élément mis en relief n'est pas ici le thème, élément connu de la phrase, mais le rhème, un élément nouveau. Ainsi le clivage et la dislocation (qui extrait hors du cadre de la phrase le thème) assurent-ils deux rôles communicatifs différents.

61 Le clivage du français, tout en situant un constituant en tête de phrase, sert donc à mettre en relief le rhème de la phrase. En finnois, la place du rhème est principalement à la fin de la phrase, dans le champ final (loppukenttä) (ISK, p. 1320-1321) ${ }^{10}$. Notre corpus 
français contient six occurrences avec une clivée, et il est clair que le rhème mis en relief par cette construction ne pourrait se trouver en tête de phrase en finnois :

(31) Cécile Duflot a pris son temps avant de répondre à l'« Appel du 22 mars » de Daniel CohnBendit. Dans un courrier qu'elle adresse, lundi 12 avril, à tous les militants Verts, comme à ceux d'Europe Écologie, la secrétaire nationale des Verts donne sa vision de l'avenir des écologistes. Elle prend le contre-pied du député européen qui préconise la structuration rapide d'Europe Écologie et le dépassement des Verts, mais ne le désavoue pas complètement. [alinéa] Première différence : la façon d'entrer dans le débat. C'est par une « lettre personnelle » adressée aux militants qu'elle fait ses propositions, en précisant... (Duflot)

En finnois le constituant clivé ne pourrait se mettre qu'à la place du rhème dans le champ final de la phrase (31'). (31") serait impossible du point de vue de l'organisation informationnelle de la phrase :

(31') [trad. par nous] Ensimmäinen ero on tapa, jolla hän avaa keskustelun. Hän esittää ehdotuksensa aktivisteille lähettämänsä kirjeen välityksellä...

(31”) ? ? Aktivisteille lähettämänsä kirjeen välityksellä hän esittää ehdotuksensa...

L'exemple suivant est tiré d'un début d'article :

(32) C'est aux côtés des personnels de santé en lutte contre le « démantèlement » de l'hôpital public qu'Eva Joly a démarré sa course électorale, vendredi 14 janvier. Pas encore désignée candidate écologiste à l'élection présidentielle de 2012, concurrencée par Nicolas Hulot qui hésite, mais distille ses envies de se présenter, la députée européenne n'a pas la tâche facile. Elle compte faire la différence en allant sur le terrain, à la « rencontre du réel ». (Joly 2)

Ici aussi, le constituant clivé ne pourrait se trouver qu'au champ final en finnois :

(32') [trad. par nous] Eva Joly aloitti kampanjansa perjantaina 14. tammikuuta julkisen sairaalan alasajoa vastustavan henkilökunnan kanssa...

\subsection{Motivation 2 : Nouveau cadre pour la phrase}

Il arrive aussi que l'adverbial initial n'établisse pas de lien référentiel avec le contexte gauche, mais qu'il mette en place un nouveau "cadre de référence " au sein d'un énoncé (cf. l'exemple 18 ci-dessus). Dans ce cas, il n'y a pas de continuité thématique au sein du texte, mais un nouveau thème de nature adverbiale est posé dans l'énoncé et le seul fait de le mettre en tête de phrase en fait un cadre pour la phrase. Ce type d'emploi des adverbiaux est mentionné dans plusieurs grammaires, aussi bien de français que de finnois. Riegel et al. parlent de circonstants à fonction scénique, qui " participent à la mise en place préalable du cadre de circonstances ou de connaissances thématisées où se situe le reste de la phrase » $(2009$, p. 266). Pour le finnois, ISK $(2004$, \$962) emploie le terme puite- ou kehysadverbiaali, "adverbial de cadre", qui remplit cette même fonction. Selon les termes de Charolles (2003, p. 29), le rôle d'un adverbial situé en tête de phrase est d'indexer la phrase en tête de laquelle il apparaît, c'est-à-dire de sélectionner un critère concernant ses circonstances d'existence qui agit sur la phrase entière (ou même au-delà, cf. motivation 3). Ce type de motivation est relativement fréquent, notamment avec les adverbiaux spatio-temporels. Dans notre corpus, cette situation a lieu environ dans un tiers des cas, et elle est plus fréquente en finnois qu'en français.

Les cas les plus évidents où l'adverbial initial n'entretient pas de lien référentiel avec le contexte gauche, fréquents selon Charolles (2003, p. 28), sont ceux où ils sont dans des 
phrases qui commencent un texte. Dans la partie finnoise du corpus, nous trouvons l'occurrence suivante qui commence l'article :

(33) Ranskan äärioikeiston johdossa tapahtui sukupolvenvaihdos sunnuntaina. Kansallisen rintaman (FN) uudeksi johtajaksi valittiin Marine Le Pen, 42 . Hän on puoluetta liki 40 vuotta johtaneen Jean-Marie Le Penin, 82, nuorin tytär. (Le Pen 2)

[trad. Dans la direction de l'extrême droite française, il y a eu un changement de génération dimanche. On a élu comme nouvelle secrétaire générale du parti du Front National (FN) Marine Le Pen, 42 ans, la fille cadette de Jean-Marie Le Pen, qui a dirigé le parti durant presque quatre décennies.]

Cependant, c'est l'unique occurrence de ce type dans notre corpus, ce qui s'explique sans doute par la nature de celui-là: étant constitué d'articles concernant des personnages connus du grand public, chaque article comporte dès le début au moins un élément qui est déjà familier pour le lecteur. Il est ainsi naturel de commencer l'article, selon les règles discursives (qui disent de commencer une séquence par quelque chose de connu), justement par cet élément connu du discours. Il devient dès lors inutile de poser comme thème une description cadrative temporelle ou locative, qui, en tant qu'éléments génériques, sont toujours potentiellement connus des lecteurs - tout événement a lieu dans un certain lieu à un certain moment -, mais qui constituent toutefois des thèmes par défaut, utilisés s'il n'y a pas de thème plus connu à disposition (Charolles 2003, p. 29). Des thèmes spatio-temporels sont sélectionnés surtout avec les faits divers, où la situation de départ est entièrement inconnue du public. Dans ce cas, des descriptions cadratives temporelles et locatives permettent de donner des éléments au moins un peu familiers au lecteur.

Cela dit, nous pouvons faire l'hypothèse que dans l'exemple (33) il s'agit justement d'événements moins familiers au lecteur finlandais et que pour cette raison il faut donc créer le cadre pour ce qui suit ${ }^{11}$.

Mais des adverbiaux-cadres se trouvent aussi ailleurs qu'en début de texte. Dans l'exemple suivant, les adverbiaux ne reprennent rien dans le discours précédent : ici, il s'agit d'un paragraphe à l'intérieur du texte où on commence à parler de la vie présente d'Éva Joly. Le paragraphe précédent est consacré à la description de l'orgueil d'Éva Joly et ne dit rien sur ses occupations.

(34) L'orgueil, dissimulé derrière une timidité apparente, est palpable. Cet ego - qui lui avait attiré le surnom d"“Eva la diva" chez certains de ses collègues de la magistrature exaspérés par sa certitude d'avoir toujours raison - semble son armure. Il la fait tenir depuis des années et ce sera probablement le moteur le plus puissant de sa décision. [alinéa] Pour le moment, elle donne le change, travaille, est totalement investie dans sa mission de députée européenne et de présidente de la commission développement. Dans son bureau à Bruxelles - derrière la porte numérotée

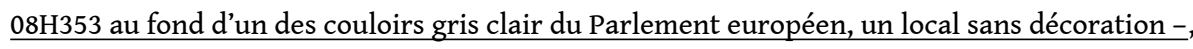
elle raconte son "immense plaisir" à siéger. Pour elle, Européenne entre deux cultures, le parlement a un rôle essentiel. (Joly 1)

70 La majorité des adverbiaux jugés comme cadres sont spatio-temporels, mais il y en a aussi d'autres types. Dans l'exemple suivant qui parle de Kadhafi, l'adverbial cadratif a un sens plutôt causatif :

(35) Pourtant, il se brouillera rapidement avec tous ses voisins arabes, au point de ne même pas être consulté lors de la guerre du Kippour contre Israël, en octobre 1973. Fort des ressources pétrolières de son pays, il se fait le champion d'une « révolution culturelle » théorisée dans un Livre vert publié en 1976, prônant une troisième voie entre socialisme et capitalisme. (Kadhafi 2) 
71 En finnois, les adverbiaux cadratifs spatio-temporels (36) sont également fréquents, mais les autres types $(37,38)$ le sont encore plus. Dans aucun des exemples suivants il n'a été question auparavant dans le texte de l'entité dont parle l'adverbial :

(36) Maailmalla Gaddafin puheeseen reagoitiin huolestuneesti. (Gaddafi 2) [trad. Dans le monde, le discours de Kadhafi a suscité de l'inquiétude]

(37) "Ääriliberalismilla on rajoitteensa. Kansalaisten kannalta tiukat säännöt ovat aina parempia", Joly sanoo. (Joly 2) [trad. « Le libéralisme extrémiste a ses désavantages. Du point de vue des citoyens, les règles strictes sont toujours meilleures ", dit Mme Joly.]

(38) Valtiona Libya eroaa suuresti Egyptistä ja Tunisiasta, joissa yhtenäinen kansallistunto antoi voimaa vastalauseliikkeelle ja auttoi myös viemään asevoimat vähitellen mielenosoittajien rinnalle. (Gaddafi 1) [trad. En tant qu'État, la Libye diffère beaucoup de l'Égypte et de la Tunisie, où un sentiment national unifié a renforcé le mouvement de révolte...]

Dans l'emploi des adverbiaux cadratifs sans relation avec le contexte gauche, on constate une différence de comportement entre le finnois et le français : en plus du fait qu'ils sont plus nombreux en finnois qu'en français (environ un tiers des occurrences contre un quart environ), on trouve en finnois davantage d'adverbiaux initiaux, autres que spatio-temporels, dont la seule fonction est de mettre en place un cadre uniquement pour la phrase, alors qu'en français, les adverbiaux initiaux cadratifs ont plus tendance à indexer la phrase en ayant simultanément une portée plus large vers la droite du texte (cf. motivation 3 ci-dessous).

\subsection{Motivation 3 : Portée vers la droite dans le texte}

Nous avons déjà vu que les adverbiaux initiaux fixent un cadre pour l'interprétation de la proposition en tête de laquelle ils se trouvent, mais cette fonction peut être destinée à continuer au-delà de la seule phrase (Virtanen 1992, 2004 et, pour une référence ponctuelle, Charolles 2003, p. 39). Un adverbial initial sert alors de cadre ou d'arrièreplan pour l'interprétation de ce qui suit ; il peut potentiellement étendre son influence à une ou plusieurs phrases qui suivent. Les interlocuteurs (les lecteurs) gardent en mémoire ce cadre pour interpréter la suite du texte. Nous pouvons parler d'une portée cadrative de l'adverbial initial. Ce sont en particulier les adverbiaux temporels et spatiaux qui ont un potentiel cadratif, mais ce ne sont pas les seuls (Charolles \& Vigier 2005, p. 12).

74 Charolles propose (2003, p. 29) que l'adverbial situé en tête de phrase a un rôle pour indexer la proposition en tête de laquelle il apparaît. Lorsque ce critère sélectionné est générique (temps ou lieu, cf. ex. 39), la décision consistant à l'exploiter comme un index n'a pas besoin d'être justifiée par le contexte précédent. Mais lorsque le critère retenu est moins prédictible, on s'attend par contre à ce que son choix soit motivé par le contexte.

(39) Pääkaupunki Tripolissa johtajan kuvat tuijottavat kansaa liikenneympyröissä ja

hallintorakennusten seinillä. Ulkomaalaisia vieraita Gaddafi ottaa vastaan luksusteltassaan, jossa hänen hyvinvoinnistaan huolehtivat kauniit naishenkivartijat. Jokainen heistä on neitsyt, kerrotaan. [alinéa] (Gaddafi 3) [trad. À Tripoli, la capitale, les photos du dictateur fixent du regard le peuple dans les ronds-points et sur les murs des bâtiments administratifs. Kadhafi reçoit des invités étrangers sous sa tente de luxe, où de belles femmes gardes du corps s'occupent de son bien-être. Chacune est vierge, d'après ce qu'on dit.] 
adverbiaux peuvent par exemple baliser les étapes de développement de quelque chose et sont alors utilisés en série (cf. Charolles 2003, p. 41-42). Généralement, dans cet emploi cadratif, le premier des adverbiaux cadres est précédé d'une phrase introductive. Cette phrase amorce (au début du paragraphe, par exemple) justifie le détachement en position de thème des adverbiaux cadres qui vont suivre (Charolles \& Vigier 2005, p. 11). Ceci rend la lecture du texte plus facile. On trouve facilement des exemples de ce genre en français :

(40) Isolé par les sanctions mais renforcé par la hausse des prix du pétrole, le Guide parvient à renouer avec les Américains en proposant son efficace coopération dans la lutte contre le terrorisme au lendemain du 11 septembre 2001. Après l'offensive américaine contre Saddam Hussein de 2003, Kadhafi renonce officiellement à un programme d'armes de destruction massive incluant un volet nucléaire. En parallèle, une médiation engagée, dès 1998, par Nelson Mandela a permis de livrer des suspects des attentats, puis de mettre en place un mécanisme d'indemnisation des victimes. Mais le Guide ne renoncera jamais à son chantage au pétrole. En 2009, il obtient la libération, " pour raisons médicales ", d'Abdelbaset Al-Megrahi, condamné, en 2001, à la prison à vie, en Écosse, pour sa participation à l'attentat de Lockerbie. [...] (Kadhafi 2)

La première phrase, en gras, est une phrase amorce dans cet exemple. En revanche, dans la partie finnoise du corpus, nous n'avons pas trouvé de phrases amorces.

Pourquoi donc l'adverbial initial a-t-il cette possibilité de former un cadre discursif ? Selon Charolles (2003, p.45), la réponse est justement qu'ils sont placés en tête de phrase, avant la prédication " principale ». Cette place est pragmatiquement destinée à accueillir des informations déjà évoquées (c'est la place typique du thème) ou des marqueurs de relations rhétoriques (par ex. des connecteurs). Pour sa part, Virtanen (2004) discute le côté « cognitif» de la question et constate que l'emplacement initial est effectivement un point de départ pour la distribution de l'information. L'information présentée en tête de phrase est susceptible de rester accessible jusqu'à la fin de l'unité textuelle qu'elle introduit (id. p. 95).

78 En français, la possibilité cadrative est utilisée très largement, tandis qu'en finnois le procédé est moins usité. L'exemple suivant provient encore d'un article sur Kadhafi. La première phrase est une phrase amorce, qui ouvre la situation, ensuite sont posés les cadres, souvent temporels, mais ils peuvent être d'autres types aussi :

(41) La contestation du régime libyen ne date pas d'aujourd'hui. Déjà en 1993, la puissante tribu des Warfallah (1 million de personnes environ), au centre-est du pays, s'est soulevée ; la répression avait fait 1200 morts, et de généreuses promesses avaient rétabli un semblant d'ordre. Quelques années plus tard, Kadhafi fait bombarder au napalm les combattants islamistes réfugiés dans le maquis du djebel vert, non loin de Benghazi. C'est dans cette même région qu'il échappe à un attentat en 1998. [...] (Kadhafi 4)

On pourrait parler de "sous-thèmes " qui apparaissent à côté du grand thème qui traverse le texte entier. Dans l'exemple, on parle de Kadhafi (c'est le thème), et on explique ce qu'il a fait à différentes époques de l'histoire de son pays.

Charolles et Vigier (2005, p. 13) parlent également de thèmes « de discours local ». Ce thème de discours local est annoncé dans la phrase amorce. Les adverbiaux initiaux ne participent pas (ou pas directement) à la mise en place de ce thème, mais ils ont une fonction organisatrice du texte : l'auteur choisit un élément du contenu propositionnel des énoncés pour distribuer ceux-ci dans des blocs informationnels homogènes ${ }^{12}$. 
81 Dans l'exemple suivant, le jeu des cadres ouverts par les adverbiaux est subtil (phrase amorce en gras) :

(42) Née en Norvège en 1943, elle gagne une bourse dans un concours et s'envole pour la France comme fille au pair. Elle découvre alors le milieu très coincé de la bourgeoisie parisienne $\mathrm{du} 16^{\mathrm{e}}$ arrondissement. [5 lignes de textes] Après les amphis, elle travaille son français dans un institut de phonétique : "C'était si difficile de faire le " $u$ " de "rue" ", sourit-elle. À 31 ans, elle devient conseillère juridique dans un hôpital, passe son concours de magistrate en 1981 et devient substitut du procureur à Orléans puis à Évry... En 1993, elle est nommée juge d'instruction au pôle financier : la consécration. [9 lignes de texte] Depuis elle est revenue en France, a « tourné la page de la Norvège » : « Là-bas, j'étais perçue comme une Française, je n'avais plus les codes », raconte-t-elle. Le mode de vie français lui manquait. «En Norvège, on ne négocie pas, il y a des règles et c'est tout. $[. .] ».($ Joly 1$)$

82 Les expressions cadratives qui structurent le texte peuvent se hiérarchiser comme ici : par exemple, on peut avoir une expression dont la portée cadrative s'étend en aval à tout le paragraphe, et en même temps, il peut y avoir dans le même paragraphe d'autres expressions initiales cadratives qui sont subordonnées à la première et qui figurent donc sous son influence. Leur portée cadrative est plus restreinte (dans cet exemple : là-bas et en Norvège). La portée du cadratif temporel depuis continue jusqu'à la fin du paragraphe.

Plusieurs indices permettent de reconnaître que la portée du cadre continue ou se termine. Selon Charolles et Vigier (2005, p. 14-15), la portée des cadres a tendance à continuer jusqu'à ce qu'il y ait un indice qui en marque la fin. Ce peut être par exemple un nouveau cadre ou l'alinéa et le changement de thème de discours.

84 En ce qui concerne la partie finnoise du corpus, nous pouvons considérer qu'à peu près un cinquième de toutes les occurrences témoigne d'une portée cadrative (les deux occurrences de välillä, 'parfois', fonctionnent dans la deuxième phrase en tant qu'expressions cadratives subordonnées à poliitikkona) :

(43) Poliitikkona hän on äärimmäinen selviytyjä, joka oppi sinnikkääksi ja taitavaksi jo nuorena.

[alinéa] Välillä se on tarkoittanut tukea terroristeille, välillä heidän vastustajilleen. Suosiota ja liittolaisuuksia hän on ostanut öljy- ja kaasurahoilla. [alinéa] Gaddafin yllättävä päätös luopua ydinaseohjelmasta vuonna 2003 pelasti Libyan suhteet länteen ja tasoitti tietä diktatuurin jatkumiselle. [alinéa] Libyan kansannousu on osoittanut, ettei Gaddafi ole valmis luovuttamaan nytkään. [alinéa] Nyt Libyan yössä [nouveau cadre] eivät kaiu vain hajanaiset laukaukset. [...]

(Gaddafi 3) [trad. En tant que politicien, c'est un vainqueur qui a appris la persévérance et l'habileté dès son jeune âge. [alinéa] Parfois cela a signifié son soutien aux terroristes, et parfois à leurs adversaires. Il s'est acheté des faveurs et des alliances avec l'argent du pétrole et du gaz. [alinéa] La décision inattendue de Kadhafi de renoncer à son programme nucléaire en 2003 a sauvé les relations de la Libye avec l'Occident et a permis à Kadhafi d'asseoir sa dictature. [alinéa] La révolte de la Libye a montré que Kadhafi n'est toujours pas prêt à renoncer à son pouvoir. [alinéa] Maintenant dans la nuit libyenne [nouveau cadre] ne retentissent pas uniquement des coups de feu isolés.]

Contrairement au français, en finnois, la portée cadrative d'un adverbial ne se termine pas forcément à l'alinéa, comme le montre l'exemple (43). Ce qui est également intéressant d'un point de vue contrastif, c'est qu'en français les adverbiaux ont tendance à être organisés en séries selon le type de l'adverbial: par exemple, adverbiaux temporels, puis spatiaux, mais les mélanges des deux types ne sont pas utilisés. En revanche, en finnois, on peut avoir des suites d'adverbiaux plus hétérogènes (voir motivation 4). 


\subsection{Motivation $4:$ « Moule » textuel} même phénomène peut se produire en finnois, mais sans que les adverbiaux ne forment une série, c'est-à-dire que la simple présence d'un adverbial initial suffit sans qu'il soit nécessaire que son type reste le même. Il peut alors être constaté qu'un adverbial est répétitivement mis en position initiale dans la phrase, et cette configuration forme une sorte de moule pour créer la cohésion textuelle. Les exemples finnois suivants illustrent ce phénomène :

(44) Joly ei ole kuitenkaan niin vanha, etteikö hänestä puuhattaisi Ranskan vihreiden presidenttiehdokasta. [alinéa] Ehdokkuudesta päätetään vuoden kuluttua syksyllä. [alinéa] "Meillä vihreillä on aivan uusi poliittis-ekologinen viesti. Meille talouskasvu ei ole kaikki kaikessa", Joly kertoo. [alinéa] Kuin ohimennen hän lyttää Ranskan nykyisen presidentin Nicolas Sarkozyn. [alinéa] "Sarkozylla oli vakavia ongelmia jo ennen valintaa. Missään muualla häntä ei olisi valittu lainkaan presidentiksi." [alinéa] (Joly 2) [trad. M ${ }^{\text {me }}$ Joly n'est toutefois pas si âgée qu'on n'essaie pas de faire d'elle la candidate aux présidentielles des Verts. [alinéa] Il va être décidé de la candidature en automne dans un an. [alinéa] « Nous les Verts avons un nouveau message politico-écologiste. Pour nous, la croissance économique n'est pas la seule chose qui compte », dit Éva Joly. [alinéa] Comme en passant, elle descend l'actuel président Nicolas Sarkozy. [alinéa] « Sarkozy avait de sérieux problèmes déjà avant d'être élu. Nulle part ailleurs il n'aurait été élu président. » [alinéa]]

Toutes les phrases commencent par un adverbial. Cette caractéristique semble jouer pour la cohésion stylistique, et en dernier lieu, textuelle du texte ${ }^{13}$. L'exemple suivant illustre le même phénomène :

(45) Aggressiivisessa puheessaan Gaddafi uhkasi nujertaa mielenosoittajat kuin torakat. Uhkaus oli pelästyttänyt mielenosoittajat. [alinéa] Tähän mennessä Gaddafi on ulkoistanut väkivallan ilmeisesti Nigeriasta ja Tšadista tulleille palkkasotureille. Puheen pelättiin merkitsevän, että Gaddafi ottaisi käyttöön armeijan erikoisjoukot. [alinéa] Toisaalta kotiin jäämisestä tuli keino jatkaa mielenosoituksia. Hallitus oli BBC :n mukaan lähettänyt keskiviikkona tekstiviestejä, joissa kehotettiin viranomaisia ja muita työntekijöitä palaamaan töihinsä. [alinéa] "Näin voimme jatkaa mielenosoituksia - aiomme pysyä kodeissamme ikuisesti”, eräs tripolilainen sanoi BBC :lle. [alinéa] Tripoliin oli lähes mahdoton saada yhteyttä edes Libyan sisältä, kertoi 28-vuotias libyalaismies HS :lle. Hän oli tullut Suomeen puolitoista viikkoa sitten. [alinéa] (Gaddafi 4) [trad. Dans son discours agressif, Kadhafi a menacé d'écraser les manifestants comme des cafards. La menace avait fait peur aux manifestants. [alinéa] Jusqu'à maintenant, Kadhafi avait fait exécuter la répression par des mercenaires venus probablement du Niger ou du Tchad. Le discours a fait craindre que Kadhafi n'ait recours aux forces de l'armée. [alinéa] D’autre part, le fait de ne pas aller travailler est devenu une façon de continuer les manifestations. Selon la BBC, le gouvernement avait envoyé mercredi des SMS incitant les autorités à rejoindre leur lieu de travail. [alinéa] « De cette façon nous pouvons continuer les manifestations - nous ne sortirons plus jamais de chez nous ", a dit un habitant de Tripoli à la BBC. [alinéa] Tripoli était presque injoignable même depuis la Libye même, a dit à Helsingin Sanomat un Libyen de 28 ans, en Finlande depuis une semaine et demie.]

88

Le corpus français ne comporte pas de passages de cette sorte: il semble que cette façon de commencer ainsi les phrases répétitivement par un adverbial soit un procédé stylistique propre à la langue finnoise. 
Notons cependant que cette quatrième motivation selon laquelle la configuration de la phrase se reproduirait et formerait un moule pour créer la cohésion textuelle reste une ébauche. Notre corpus est trop restreint pour avancer des analyses définitives.

\section{Conclusion}

Les observations et analyses faites dans cette étude concernent les textes journalistiques. Notre hypothèse de départ n'est pas confirmée : les adverbiaux initiaux ne sont pas nettement plus fréquents dans les textes de presse en français qu'en finnois. On aimerait plutôt dire le contraire. Mais ce qui est intéressant, c'est que leurs fonctions sont différentes dans les deux langues.

91 Le français semble tirer profit de la possibilité de placer le circonstant en tête de proposition pour des raisons textuelles : la position initiale est volontiers motivée par la structure du texte au-delà de la phrase.

En finnois en revanche, c'est surtout la structure informationnelle à l'intérieur de la phrase qui place l'adverbial au début; dans les phrases de type spécifique, les phrases existentielles par exemple, l'adverbial initial se place dans la position de thème - dans ces phrases cette position de l'adverbial est non marquée.

En généralisant un peu, dans le cadre du texte, l'adverbial en tête de proposition a une fonction locale en finnois, mais globale en français.

\section{BIBLIOGRAPHIE}

CHAROLLES Michel, 2003, « De la topicalité des adverbiaux détachés en tête de phrase », in CHAROLles Michel \& PREVOST Sophie (éd.), Adverbiaux et topiques, Travaux de linguistique 47, p. 11-49.

CHAROLLES Michel \& VIGIER Denis, 2005, « Les adverbiaux en position préverbale : portée cadrative et organisation des discours ", in Charolles Michel \& Péry-Woodley Marie-Paule (éd.), Les adverbiaux cadratifs, Langue française 148, p. 9-30.

COMBETTES Bernard, 1998, Les constructions détachées en français, Paris : Ophrys.

COMBETTES Bernard, 2005, « Les constructions détachées comme cadres de discours », in CHAROLLES Michel \& PERY-WOODLEY Marie-Paule (éd.), Les adverbiaux cadratifs, Langue française 148, p. 31-44. DUVALLON Outi, 2003, «L'ordre sujet-complément-verbe dans les textes oraux en finnois ", Études finno-ougriennes 35, p. 131-160.

DUVALLON Outi, 2011, Le modèle positionnel proposé par M. Vilkuna pour l'analyse de l'ordre des mots en finnois, Manuscrit distribué au Séminaire d'analyse contrastive des langues fenniques et du français, Institut national des langues et civilisations orientales, Paris. 
FOURNIER Nathalie, 1997, « La place du sujet nominal dans les phrases à complément prépositionnel initial », in FUCHS Catherine (éd.), La place du sujet en français contemporain, Champs linguistiques, Louvain-la-Neuve : Duculot, p. 97-132.

FUCHS Catherine \& FOURNIER Nathalie, 2003, « Du rôle cadratif des compléments localisants initiaux selon la position du sujet nominal », in CHAROLLES Michel \& PREVOST Sophie (éd.), Adverbiaux et topiques, Travaux de linguistique 47, p. 79-110.

HAKULINEN Soili \& TURKKA Pieta, (à paraître), « La cohésion discursive et la construction de l'information en contraste : la reprise d'un référent humain central dans des textes journalistiques français et finnois ", Neuphilologische Mitteilungen.

HAKULINEN Auli, vilKUnA Maria, KORHONEN Riitta, KoIVISTO Vesa, HEINONEN Tarja Riitta \& Alho Irja, 2004, Iso suomen kielioppi, Helsinki : Suomalaisen kirjallisuuden seura. [ = ISK 2004.]

ISK 2004 = HAKULINEN et al. 2004.

REMI-GIRAUD Sylvianne, 1998, « Le complément circonstanciel : problèmes de définition », in REMIGIRAUD Sylvianne \& ROMAN André (éd.), Autour du circonstant, Lyon : Presses universitaires de Lyon, p. 65-113.

RIEGEL Martin, PELLAT Jean-Christophe \& Rioul René, 2009, Grammaire méthodique du français. PUF, Paris. [ $4^{\mathrm{e}}$ édition entièrement revue « Quadrige ».]

VÄLIKANGAS Olli, 1984, « Les compléments circonstanciels initiaux dans les propositions finnoises et françaises ", Études finno-ougriennes XVIII, p. 236-257.

VILKUNA Maria, 1989, Free Word Order in Finnish: Its Syntax and Discourse Functions, Helsinki: Suomalaisen kirjallisuuden seura.

VIRTANEN Tuija, 1992, Discourse Functions of Adverbial Placement in English: Clause-Initial Adverbials of Time and Place in Narratives and Procedural Place Descriptions, Turku: Åbo Akademi University Press. VIRTANEN Tuija, 2004, « Point of departure: Cognitive aspects of sentence-initial adverbs », in VIRTANEN Tuija (éd.), Approaches to Cognition through Text and Discourse, Trends in Linguistics 147, Berlin - New York: Mouton de Gruyter, p. 79-97.

\section{NOTES}

1. Fred Vargas: Dans les bois éternels (Viviane Hamy, s.l. 2006) et sa traduction Ikimetsän sydän par Maria Luoma (Gummerus, Helsinki 2010; $2^{\mathrm{e}}$ éd., $1^{\mathrm{e}}$ éd. 2008) ; Sofi Oksanen : Puhdistus (WSOY, Helsinki $2010 ; 28^{\mathrm{e}}$ édition, $1^{\mathrm{e}}$ éd. 2008) et sa traduction Purge par Sébastien Cagnoli (Stock, s.l. 2010).

2. Nous remercions les auteurs de cette étude de la possibilité d'utiliser le même corpus.

3. Le problème avec le terme complément est qu'il ne permet pas de savoir s'il s'agit d'un constituant argumental ou non. C'est pourquoi la tradition générative distingue entre les compléments, constituants appelés par une tête de syntagme, et les modifieurs, constituants facultatifs pas appelés. Pour les compléments du verbe, la théorie valencielle emploie les termes actant et circonstant.

4. Si l'on s'en tient à des critères sémantiques, il est difficile d'établir la distinction entre un "vrai » circonstant et un "complément» (=modifieur) du verbe non 
argumental (lui aussi mobile et supprimable), c'est-à-dire de savoir si un constituant est situé au niveau du SV ou de la phrase. Selon Rémi-Giraud (1998, pp. 82-83), le critère est purement positionnel : si ce complément est placé en position initiale ou en position préverbale, il se trouve hors du champ verbal et doit être considéré comme un complément de phrase, alors qu'en position postverbale et finale, il se trouve dans la zone d'influence du verbe et il s'agit d'un complément de verbe. Dans ce sens, tous les circonstants initiaux sont des compléments de phrase.

5. Les cas grammaticaux sont considérés comme étant directement dictés par la configuration grammaticale de la phrase, tandis que pour les cas sémantiques, le cas apporte une signification particulière déjà en soi.

6. En plus des caractéristiques déjà discutées, ces éléments placés en tête de phrase ont en commun un certain pouvoir prédicatif, qui s'observe le plus clairement dans le cas de la prédication seconde mais qui se voit également dans la présence de la préposition ou du suffixe casuel en finnois, ou d'un adverbe. L'adverbial initial instaure alors une relation de prédication qui sera complétée plus tard dans la phrase. Ainsi, dans l'exemple (7), pour quelques-uns crée une relation entre le référent du SN (quelques-uns) et un autre élément qui sera révélé plus tard dans la phrase (tout est permis). Nous remercions pour cette remarque pertinente notre relecteur anonyme. Voir à ce sujet aussi Charolles et Vigier (2005, p 11).

7. Fuchs et Fournier (2003) ont montré que dans les phrases à sujet postposé, le comportement discursif des adverbiaux initiaux n'était pas le même que si le sujet était antéposé.

8. Il peut y avoir plusieurs éléments thématiques dans une phrase.

9. En contraste avec ce qui s'est passé auparavant et dont parle le contexte gauche.

10. Un syntagme préverbal seul peut parfois s'interpréter comme un rhème en finnois, par exemple lorsque le pronom sujet n'est pas réalisé (Todella tiukassa tilanteessa olemme, trad. littérale Dans une situation très difficile [nous] sommes) ou lorsque ce syntagme préverbal est le sujet (Me voitimme ettekä te, trad. C'est nous qui avons gagné, et pas vous). Mais il s'agit toujours de situations marquées (ISK 2004, p. 1320-1321).

11. Même si le titre en annonce déjà le cadre : Ranskan äärioikeiston valtikka siirtyi isältä tyttärelle, trad. Le pouvoir de l'extrême droite française est passé du père à la fille.

12. On peut, bien sûr, utiliser des adverbiaux ailleurs qu'en position initiale. Mais dans ce cas, ils n'ont pas cette possibilité de portée cadrative, et dans ce cas, si les circonstances temporelles ou autres continuent au-delà de la limite d'une phrase, l'auteur doit répéter l'adverbial ou son synonyme dans la phrase suivante (cf. ex. 3 de Charolles \& Vigier 2005, p. 13).

13. Certains des adverbiaux initiaux se trouvent ici dans des phrases «de type spécifique ». Ces phrases où le positionnement initial de l'adverbial est non marqué semblent toutefois contribuer à la formation du « moule ». 


\section{RÉSUMÉS}

Le présent article étudie les adverbiaux initiaux, c'est-à-dire en tête de proposition, dans une perspective contrastive français-finnois et avec une approche fonctionnelle. L'adverbial est ici le terme utilisé pour parler de circonstants dans un sens étendu. Nous partons de l'hypothèse que le statut des adverbiaux initiaux n'est pas identique dans les deux langues et travaillons sur un corpus de textes de presse comparable dans les deux langues. Quatre types de motivation sont relevés pour expliquer le positionnement initial de l'adverbial, tous liés à des questions thématiques : (1) l'adverbial initial entretient un lien anaphorique avec le contexte gauche et est utilisé pour créer de la cohésion textuelle ; (2) l'adverbial initial fonctionne comme un cadre pour la proposition qu'il introduit, ce que permet son emplacement au début de la proposition; (3) l'adverbial initial sert à structurer le texte (portée cadrative); (4) l'emplacement initial d'adverbiaux de différents types dans des phrases qui se succèdent forme un moule textuel qui crée de la cohésion. La comparaison des deux langues révèle qu'effectivement, le français et le finnois n'utilisent pas les adverbiaux initiaux de la même façon. En français, leur emploi pour des fins textuelles semble plus important.

The study deals with clause-initial adverbials. It compares their use in French and Finnish using a functional approach. The premise is the hypothesis that the status of clause-initial adverbials is not identical in these two languages; the corpora used consist of journalistic texts. Four different kinds of motivation for the clause-initial use of adverbials are detected, each of them related to thematic questions: (1) the clause-initial adverbial has an anaphoric relation to something in the preceding context and is used to create and maintain textual cohesion; (2) the clause-initial adverbial creates a frame for the clause it introduces, which is made possible by its placement at the beginning of said clause; (3) the clause-initial adverbial is used for structuring the text (extended frame); (4) different kinds of clause-initial adverbials are used in sentences that follow each other in the text: this is a "textual mould" that creates cohesion. The comparison of French and Finnish reveals that indeed these two languages do not use clause-initial adverbials in an identical way. In French, they are more often used for textual purposes.

Artikkelin aiheena ovat lauseenalkuiset adverbiaalit, ja sen lähestymistapa on funktionaalinen. Adverbiaaleja vertaillaan ranskassa ja suomessa. Lähtökohtana on hypoteesi, jonka mukaan lauseenalkuisten adverbiaalien käyttötapa ei ole näissä kielissä samanlainen. Tutkimuksen aineistona ovat lehtitekstit. Adverbiaalin lauseenalkuista asemaa selittämään löytyy neljä erilaista motivaatiota, jotka kaikki liittyvät teeman käsitteeseen: (1) lauseenalkuisella adverbiaalilla on anaforinen suhde edeltävään kontekstiin, ja sitä käytetään luomaan tekstuaalista koheesiota; (2) lauseenalkuinen adverbiaali toimii aloittamansa lauseen kehyksenä tai puitteena - tämän mahdollistaa nimenomaan sen lauseenalkuisuus; (3) lauseenalkuiset adverbiaalit strukturoivat tekstiä (laaja kehys); (4) tekstissä esiintyy peräkkäisissä lauseissa erityyppisiä lauseenalkuisia adverbiaaleja: tällainen tekstuaalinen muotti luo koheesiota. Ranskan ja suomen vertailu paljastaa, että ne eivät tosiaan käytä lauseenalkuisia adverbiaaleja samalla tavoin. Ranskassa niiden käyttö perustuu enemmän tekstuaalisiin syihin. 
INDEX

Thèmes : linguistique, linguistique contrastive

Keywords : Adverbials, Anaphora, Comparative Linguistics, Linguistics, Parallel Corpora, twentyfirst century, Topic, Finnish, French, World Order

Mots-clés : adverbes, anaphore, circonstants, corpus parallèles, ordre des mots, thème

Index chronologique : XXIe siècle, XXIe siècle (début)

disciplines finnois, français 